\title{
Efficient Routing by Power Optimization using Minimum Hop Count Routing Protocol in WSN
}

\author{
Farhat Afrin \\ MTech. Scholar, Department of \\ Computer Science \\ UIT, RGPV Gandhinagar \\ Bhopal, India
}

\author{
Rajeev Pandey, PhD \\ Asst. Professor, Department of \\ Computer Science \\ UIT, RGPV Gandhinagar \\ Bhopal, India
}

\author{
Uday Chourasia \\ Asst. Professor, Department of \\ Computer Science \\ UIT, RGPV Gandhinagar \\ Bhopal, India
}

\begin{abstract}
Wireless sensor networks (WSNs) take very important benefits over usual communications in today's applications like environmental observation, Homeland Security and health care. However, harsh and complicated environments pose great challenges within the reliability of WSN communications. To achieve reliable wireless communications at intervals WSNs, it's essential to own a reliable routing protocol and to own a way to evaluate the reliability performance of various routing protocols. Successive Interference Cancellation (SIC) could be a new physical layer technique that allows the receiver to decode composite signals from multiple transmitters sequentially. The introduction of sic improves the path bandwidth. During this development, the main focus is on the development of bandwidth-aware routing protocol with sic, aiming at achieving high overall end-to-end output. Throughout this paper, we tend to tend to focus on the design of bandwidthaware routing protocol with set, aiming at achieving high overall end-to-end output. A routing metric capturing the advantage of set in terms of data live and network resource is planned, by that our routing protocol can choose a path satisfying the data measure demand of this flow and reserving further network resource for the subsequent ones.
\end{abstract}

\section{Keywords}

Multihop Wireless Networks, Successive Interference Cancellation, Routing Metric, Available Bandwidth.

\section{INTRODUCTION}

In wireless multi-hop networks, nodes communicate with one another using wireless channels and do not have the requirement for common infrastructure or centralized management. Nodes may cooperate with one another by forwarding or relaying each others' packets, probably involving many intermediate relay nodes. This allows nodes that cannot hear each other on to communicate over intermediate relays while not increasing transmission power. Such multi-hop relaying may well be a really promising resolution for increasing output and providing coverage for a large physical area. By using many intermediate nodes, the sender will reduce transmission power therefore limiting interference effects and enabling spatial reprocess of frequency bands. In ad-hoc networks, the medium is shared and nodes prepare access to the medium during a distributed method independent of their current traffic demand. Especially given normal ad-hoc routing protocols that try and minimize relaying nodes on the path, nodes closer to the network centre are additional possible to become a relay node. This has the inherent disadvantage that a node that is a relay node for transmissions of multiple close nodes is prone to become a performance bottleneck. Because it is important to know performance of such relay networks, the next sub section provides a summary on performance analysis of a relay node. Once multiple relays are concerned across an endto-end path, it's necessary to manage overhead for every single packet transmission. unfortunately, current Medium Access control (MAC) and physical layers for Wireless local area Network (WLAN) based mostly multi-hop networks impose high overhead for the transmission of small information packets, that is common for voice over net Protocol (VoIP). By combining many little packets into larger ones, per packet transmission overhead will be reduced considerably.

Routing Protocols the objective of routing is to route information from a sender to 1 or additional destinations. Routing during a mobile wireless multi-hop network, and especially in mobile adhoc networks, may be a difficult task. Routing protocols in mobile ad-hoc networks are typically divided into proactive, reactive and hybrid routing. A proactive protocol evaluates routes to all accessible nodes and attempt to maintain consistent up to-date routing data. During a reactive protocol, routing methods are searched only if required. Hybrid protocols mix proactive routing with reactive routing in hierarchical network structures. The quality of nodes together of the noisy links involves new approaches so as to get optimal network performance. Also, new applications and systems need quite the normal unicast routing protocols.

In cellular and wireless local area networks, wireless communication only happens on the last link between a base station and also the wireless end system. In multi-hop wireless networks there is one or additional intermediate nodes on the path that receive and forward packets via wireless links. Multi-hop wireless networks have many benefits: Compared to networks with single wireless links, multi-hop wireless networks will extend the coverage of a network and improve property. Moreover, transmission over multiple "short" links may need less transmission power and energy than over "long" links. Moreover, they permit higher information rates leading to higher throughput and additional efficient use of the wireless medium. Multi-hop wireless networks avoid wide preparation of cables and might be deployed during a costeffective method. In case of dense multi-hop networks many methods may become available which will be used to increase robustness of the network. Unfortunately, protocols developed for fixed or cellular networks also because the internet's aren't best for multi-hop wireless networks. This is often specially the case for routing protocols, wherever completely 
new unicast, multicast, and broadcast routing protocols are developed for (mobile) ad-hoc and sensor networks.

\section{RELATED WORK}

Author [1] develops a technique to analytically compute the offered bandwidth of a given path with sic. Author additionally styles a distributed heuristic algorithmic rule so the bandwidth is calculable by a distributed routing protocol. Then, a routing metric that quantifies the advantages of set in terms of bandwidth and network resource consumption is meant. Simulations results show that the BARS explore additional sic opportunities, and so achieve vital output gain over different protocols. In this paper, [3] authors advocated a joint interference exploitation and avoidance approach, which mixes the simplest of each worlds whereas avoids each's pitfalls. We tend to discussed new challenges of such an approach in a very multi-hop wireless network and projected a formal optimization framework, with cross layer formulation of physical, link, and network layers. This framework offered a rather complete style area for sic, with the goal to squeeze the most out of interference. Authors claim that such an optimization framework is appropriate for studying a broad category of network output improvement issues. As a case study, we tend to demonstrate the way to apply such framework for a network throughout optimization problem. Our numerical results affirmed the efficacy of this framework and gave insights on the optimal interaction between interference exploitation and interference avoidance.[3] Authors tend to examine the impact of sic from initial principles and verify that a major output gain between 200th and $100 \mathrm{pc}$ is obtained from sic. Moreover, the performance gain of a scheduling scheme is basically correlated with the usage of the transmission opportunities from sic. This work demonstrates the importance of planning an SIC-aware scheduling scheme, and suggests that the approximation ratio isn't a sufficient indicator of the programming performance once sic is offered.[4] In this paper,[5] author present a highthroughput sic Aware Routing Protocol-SAR for multihop wireless networks. Authors propose a sic aware routing metric that explores the advantages related to sic in special resource consumption reduction and bandwidth efficiency improvement. Simulation results show that the SAR with success will increase sic ratio of network, thus achieves significant output gain over different protocols. The main contribution of our work may be a new left-isotonic path weight that captures the offered path bandwidth data. The leftis tonicity property of our projected path weight facilitates us to develop a proactive hop-by-hop routing protocol, and that we formally proved that our protocol satisfies the optimality and consistency needs. Based on the offered path bandwidth data, a source will immediately determine some infeasible connection requests with the high bandwidth requirement.[6]

In this paper [8] planned a routing metric iETT and a routing protocol S3 for WMNs, to need advantage of physical layer writing techniques for prime network output. iETT includes the interference-awareness property and provides a simple technique of measuring the potential gains of applying every techniques. The S3 protocol works in three steps to explore the writing opportunities. Experimental results supported antelope radio platform ensure the effectiveness of the planned protocol. Necessary enhancements in network output are determined in every single-path and multi-path routing things. In [10] proved the NP-hardness of this problem and developed integer linear programming formulations that can be used to approach the exact optimum for parallel and successive interference cancellation. Using these formulations, we have performed numerical experiments to quantitatively evaluate the gain due to interference cancellation. The simulation results indicate that for low to medium SINR thresholds, interference cancellation delivers a significant performance improvement. In particular, the optimal SIC scheme can double or even triple the number of activated links. Moreover, node density may also affect performance gains, as evidenced in one of the datasets. Given these gains and the proven computational complexity of the problem, the development of approximation algorithms or distributed solutions incorporating IC is of high relevance. Authors [11] have also shown how to leverage our technique within a distributed routing protocol which exploits local measurements performed by the nodes to effectively route newly added flows, achieving significant gains with respect to existing routing metrics. Authors plan to extend our approach to study the coupling of mesh routing protocols with dynamic data rate adjustment mechanisms such as AutoRate Fallback and congestion control protocols such as TCP. In [13] results show that WCETT outperforms previously-proposed metrics. WCETT permits us to trade off channel diversity and path length, by dynamical the value of the control parameter $\beta$. we tend to experimented with different values of this control parameter, and showed that on shorter ways, taking channel diversity into account brings vital edges. We tend to additionally show that on longer ways, yet as in heavilyloaded networks, the advantages obtained by selecting channel-diverse ways are limited.

In [15] to compute on the best throughput assume the power to finely control and thoroughly schedule packet transmissions, the best routes yielded by our analysis usually outperform shortest path routes even below |real-world" conditions like un-coordinated scheduling and mac contention. In ns-2 simulations, we've observed a turnout improvement of over an element of two in some cases. The rationale for this important improvement is that the best routes typically tend to be less interference prone than the default shortest path routes.

\section{METHOD}

\subsection{HOP COUNT ROUTING}

Hop Count Routing algorithmic rule is discovered by using HCRing or Broadcasting. Broadcasting is an inevitable operation of route discovery. Though the broadcast by HCRing is easy however inefficient and leads to redundant message relays. This successively over use of limited network resources like channel node energy and bandwidth. The traditional HCRing theme is cause high retransmissions that cause packet collisions and media congestion which will significantly degrade the network performance and output.

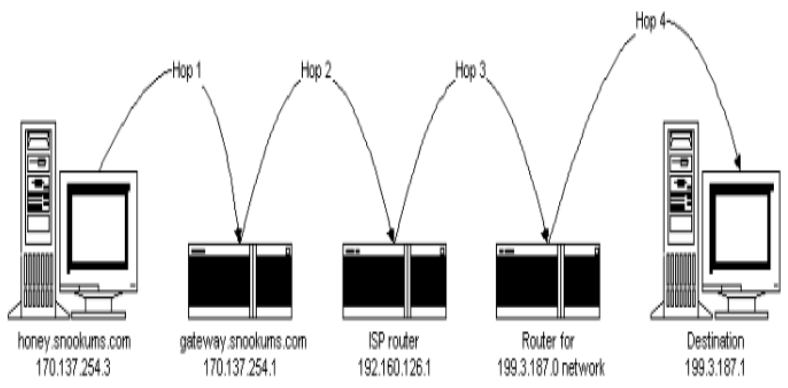

Figure 1 Hopping around 


\subsection{Multiple Route Discovery using Hop Count Routing (HCR) Algorithm}

The Multiple Route Discovery HCR algorithm implementation details are as shown below:

The following are the steps for Multiple Route Discovery using BARSIC

1. Source Node, Destination Node and Coverage area acts as an input

2. The source node routing table is fetched

3. The neighbors are discovered

4. For each of the neighbor acting like a source node. Route is discovered using individual HCR algorithm the routes are cached.

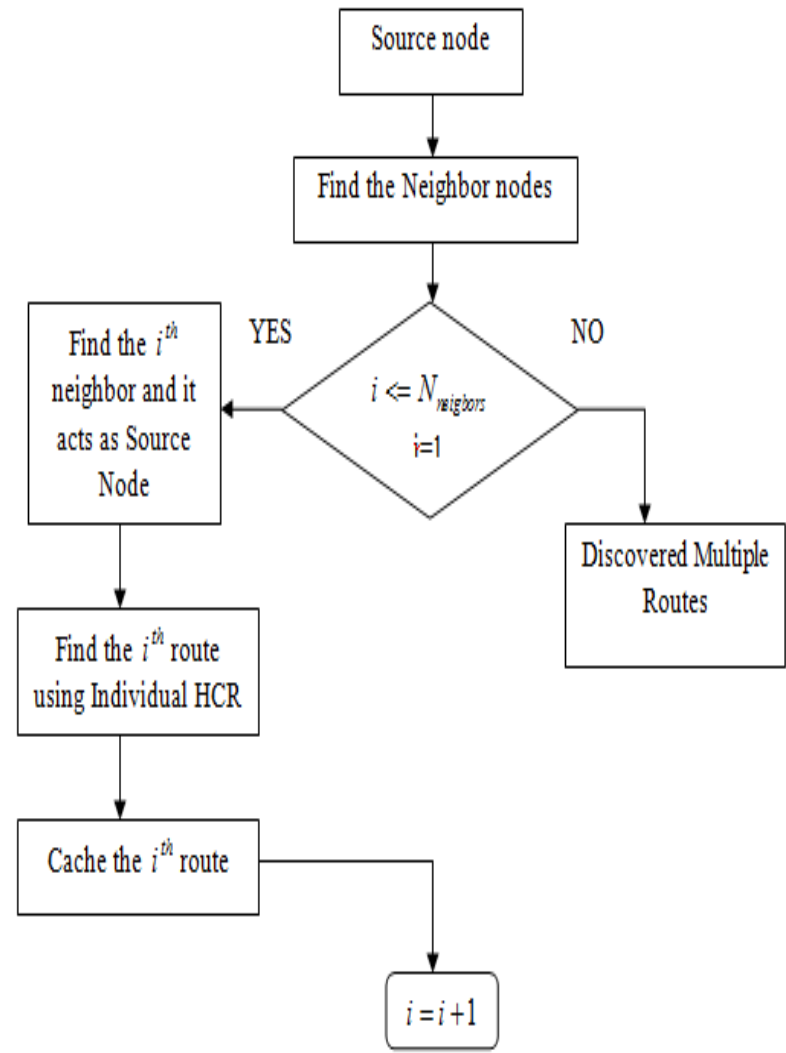

Figure 2 flow diagram of Multiple Route Discovery using BARSIC

\subsection{Individual HCR Algorithm}

Please The Individual HCR Algorithm is defined as follows:

1. Source Node, Destination Node and Transmission Range acts as an input.

2. The Source Node will find the set of nodes within transmission range known as neighbor nodes.

3. If the neighbor nodes has the destination node then stop the process.

4. If the neighbor nodes does not have the destination node then pick one of the neighbor as the next forward node which has lowest REPLY time.

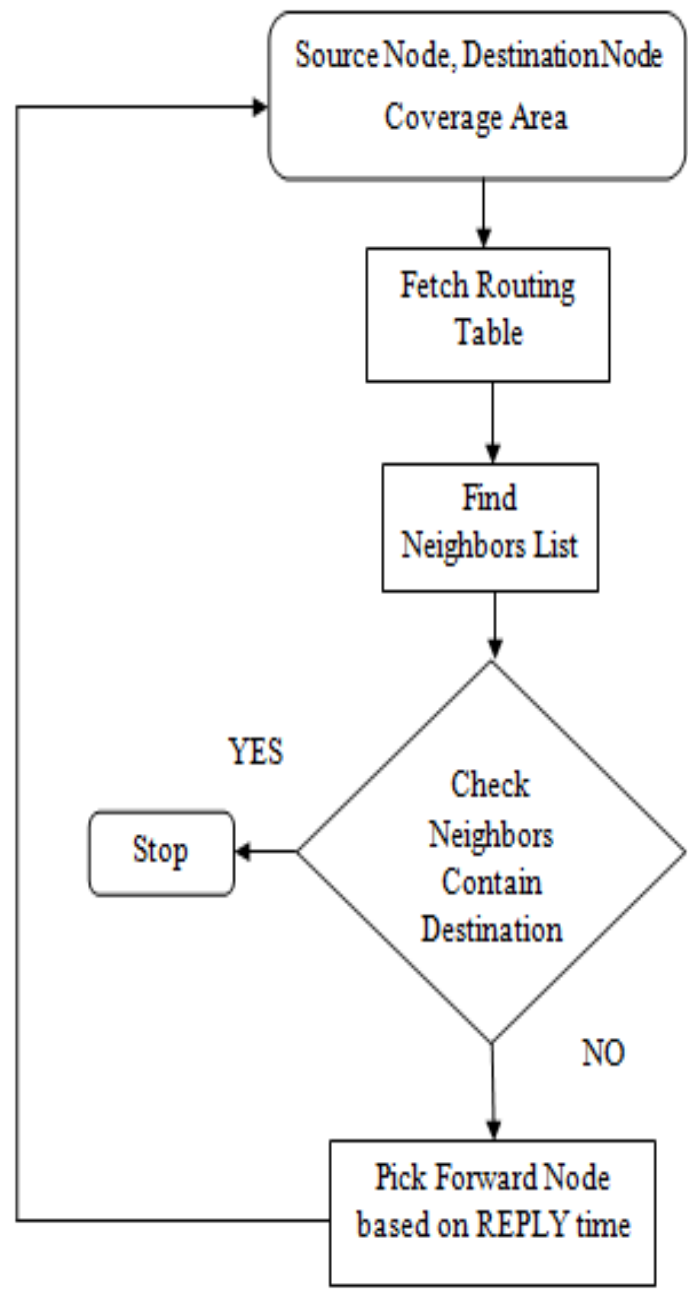

Figure 3 flow diagram of Individual HCR Algorithm

\subsection{Best Route Discovery using HCR}

1. Source Node, Destination Node and Transmission Range acts as an input.

2. The Source Node will find the set of nodes within transmission range known as neighbor nodes.

3. If the neighbor nodes has the destination node then stop the process.

4. If the neighbor nodes does not have the destination node then pick one of the neighbor as the next forward node.

5. The Multiple routes discovered using HCR acts as an input

6. For each of the route count the number of hops

7. Create a map with key as Hop Count and value as route no

8. Find the route which has lowest hop count. 


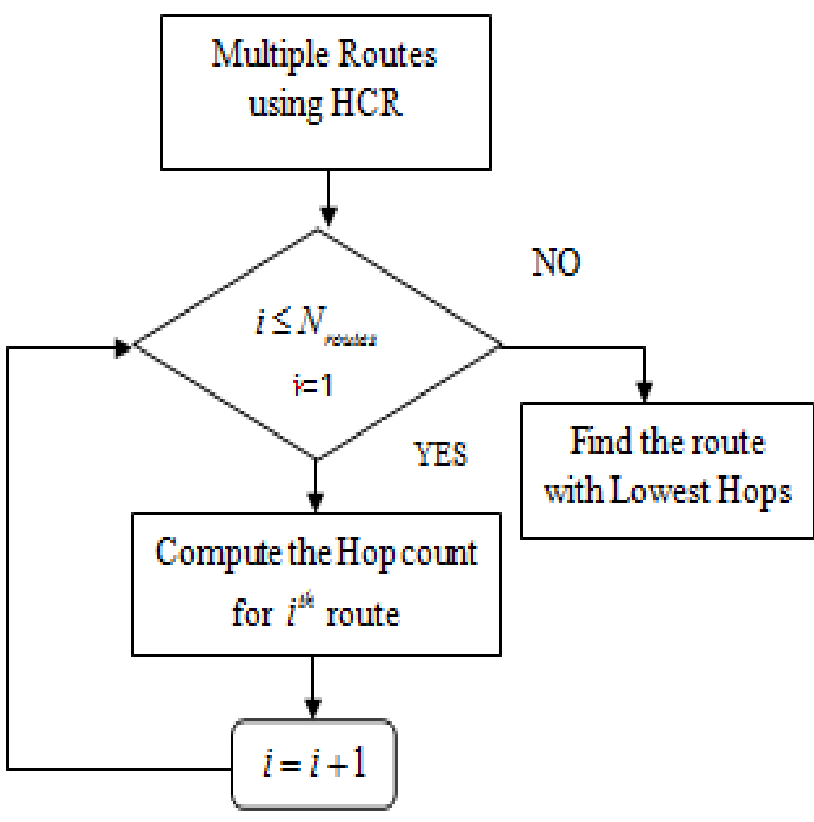

Figure 4 Best Route Discovery for HCR

\section{SIMULATION RESULTS}

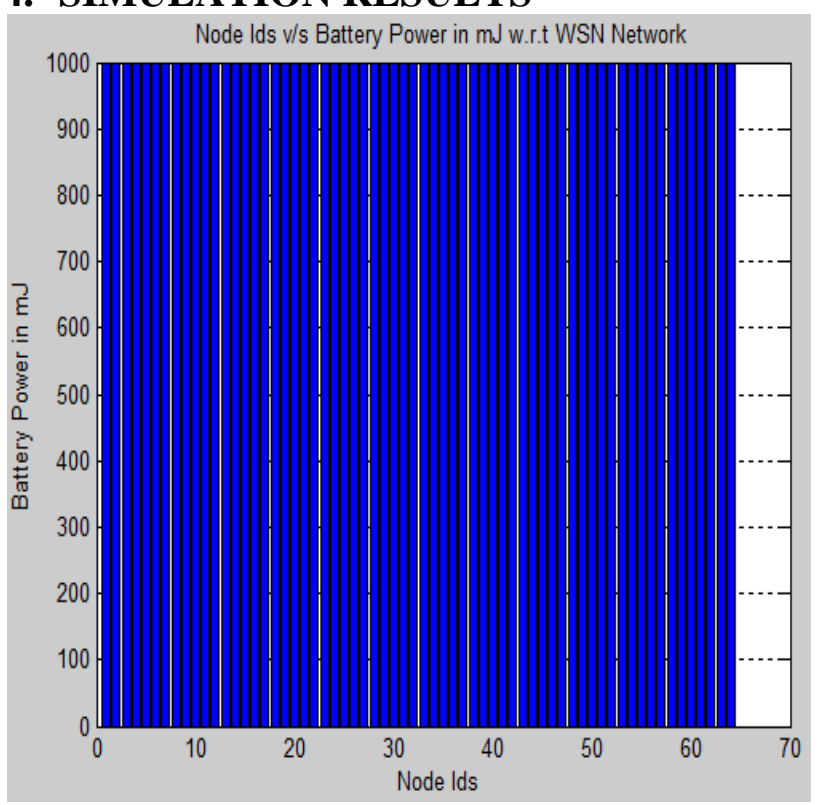

Fig.5 Node Ids v/s battery power in mj w.r.t. WSN network

This figure shows the node Ids verses battery power in $\mathrm{mj}$ with respect to WSN network. In this figure $\mathrm{X}$ level shows the Node Ids and Y level shows the battery power in mj.

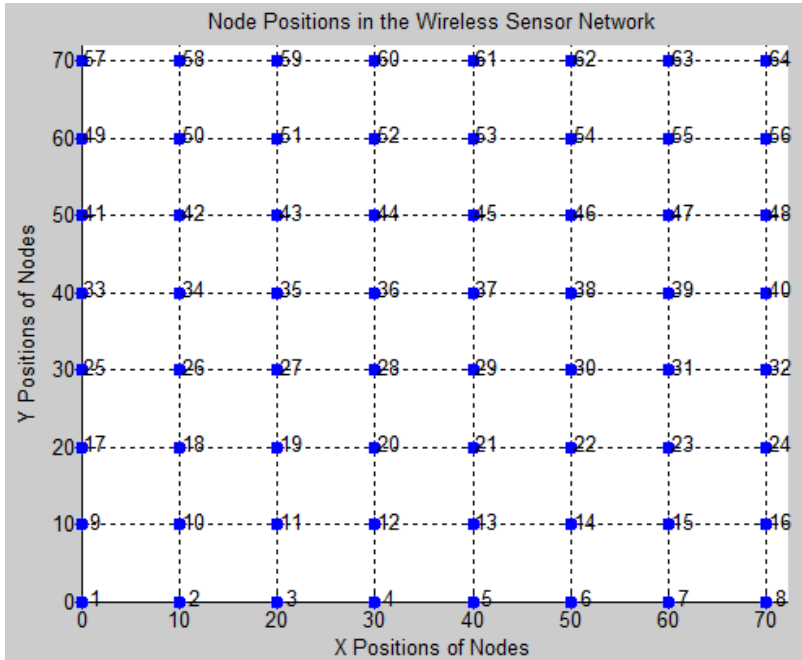

Fig.6 Node position in the wireless sensor network

This figure shows the node position in the wireless sensor network. In this figure $X$ level shows the $X$ position of nodes and $\mathrm{Y}$ level shows the $\mathrm{Y}$ position of nodes.

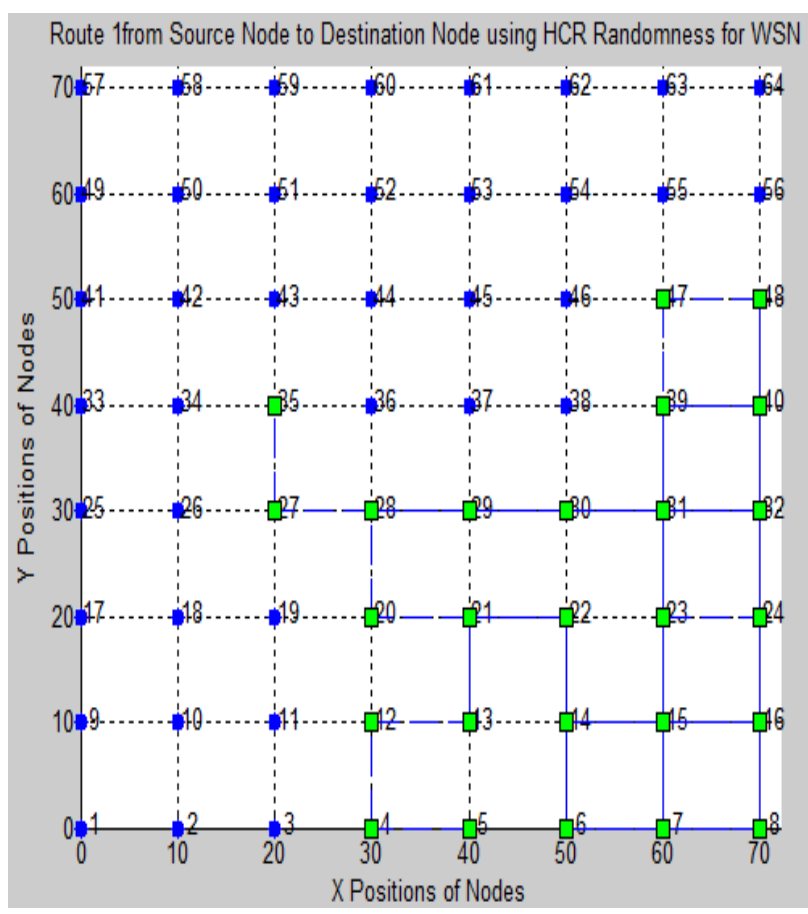

Fig.7 First route from source to destination using HCR Randomness for WSN

This figure shows the First route from source to destination using HCR Randomness for WSN. In this figure X level shows the $\mathrm{x}$ positions of nodes and $\mathrm{Y}$ level shows the $\mathrm{y}$ positions of nodes. 


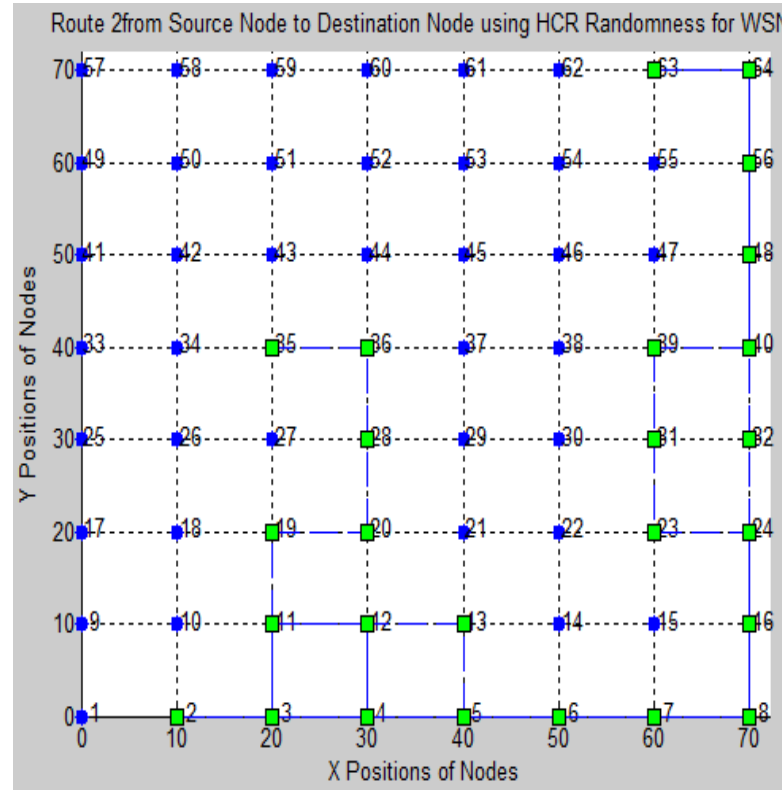

Fig.8 Second route from source to destination using HCR Randomness for WSN

This figure shows the second route from source to destination using HCR Randomness for WSN. In this figure X level shows the $\mathrm{x}$ positions of nodes and $\mathrm{Y}$ level shows the $\mathrm{y}$ positions of nodes.

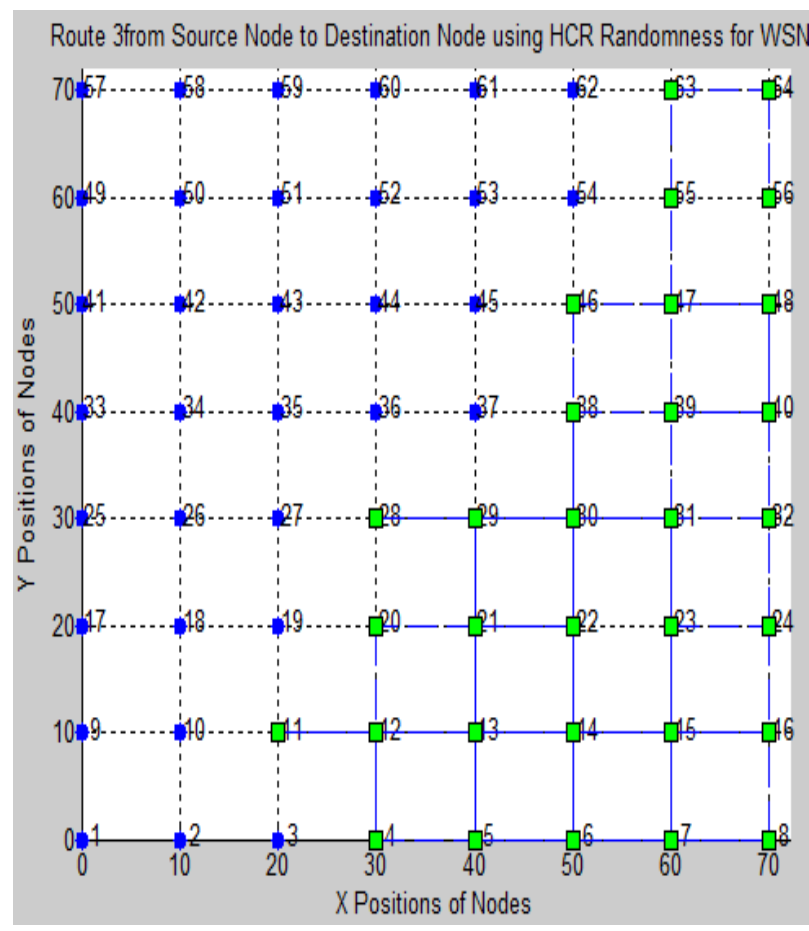

Fig.9 Third route from source to destination using HCR Randomness for WSN

This figure shows the third route from source to destination using HCR Randomness for WSN. In this figure X level shows the $\mathrm{x}$ positions of nodes and $\mathrm{Y}$ level shows the $\mathrm{y}$ positions of nodes.

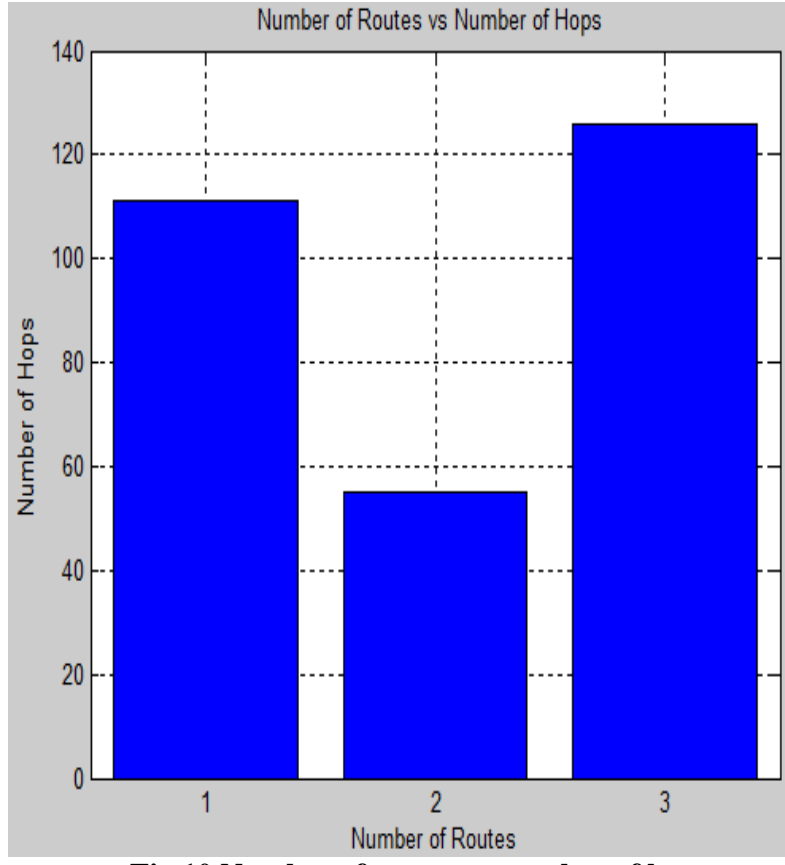

Fig.10 Number of routes vs number of hops

This figure shows the Number of routes vs number of hops. In this figure $\mathrm{X}$ level shows the number of routes and $\mathrm{Y}$ level shows the number of hops.

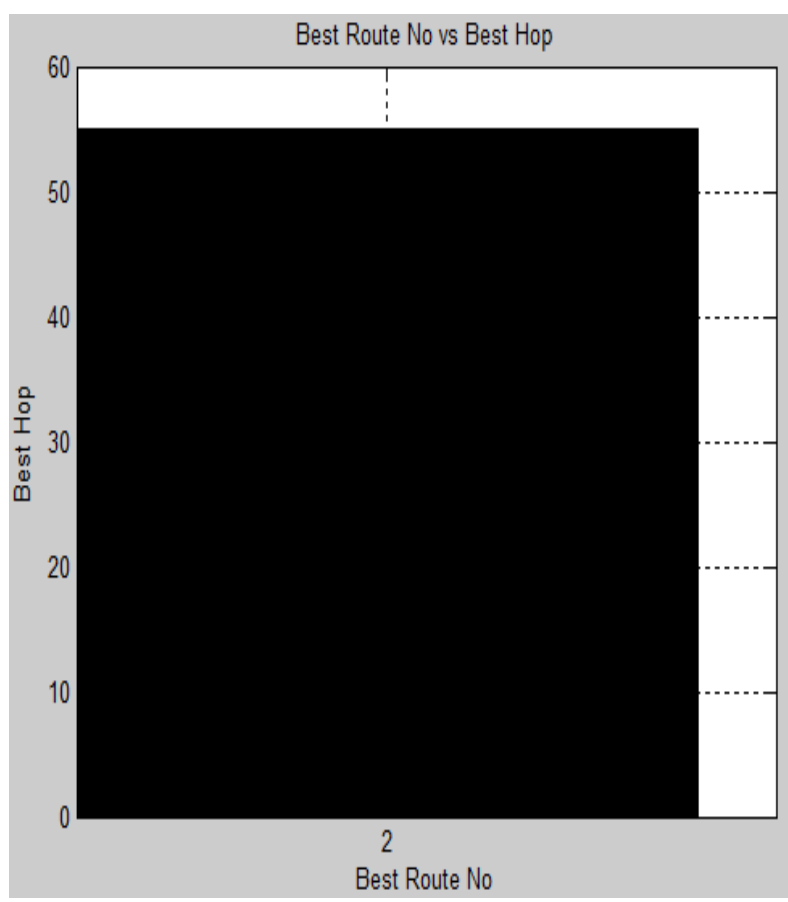

Fig.11 best route No. vs Best hop

This figure shows the best route No. vs Best hop. In this figure $\mathrm{X}$ level shows the best route No and $\mathrm{Y}$ level shows the Best hop. 


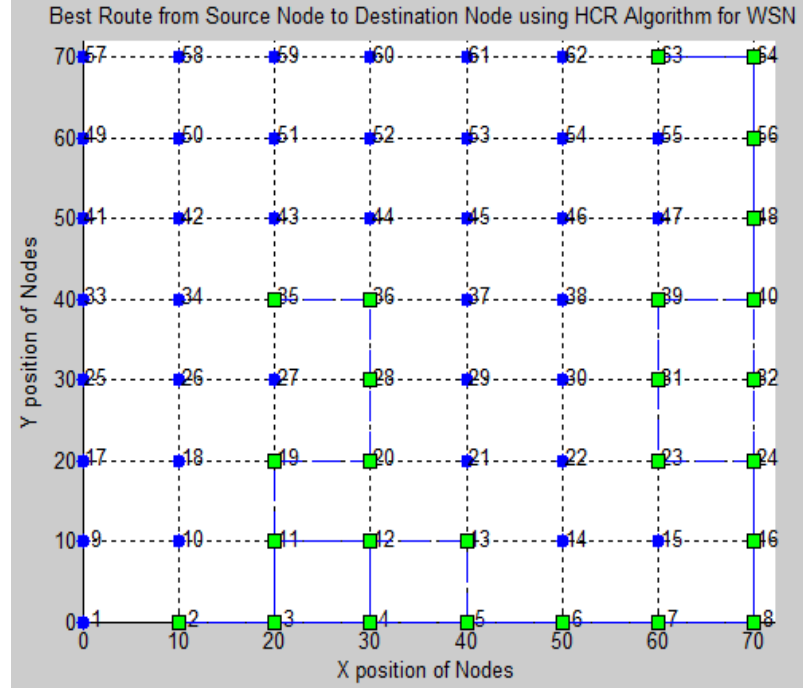

Fig.12 Best route from source to destination using HCR Randomness for WSN

This figure shows the best route from source to destination using HCR Randomness for WSN. In this figure X level shows the $\mathrm{x}$ positions of nodes and $\mathrm{Y}$ level shows the $\mathrm{y}$ positions of nodes.

\section{CONCLUSION}

This paper research we tend to present a unique routing protocol, known as dynamic minimum hoping with sic opportunities for wireless sensor networks. We tend to develop a technique to analytically calculate the offered bandwidth of a given path with sic. We tend to additionally design a hop counting algorithmic rule so the bandwidth will be estimated by a distributed routing protocol. Then, a routing metric that quantifies the advantages of sic in terms of information measure and network resource consumption is meant. Simulations results show that the HCR explore additional sic opportunities, and therefore achieve important output gain over different protocols and found the best route from source to destination using HCR randomness for wireless sensor network.

\section{REFERENCES}

[1] G. Liu, Runzi, et al. "Bandwidth-Aware HighThroughput Routing With Successive Interference Cancelation in Multihop Wireless Networks" IEEE Transactions on Vehicular Technology 64.12 (2015): 5866-5877.

[2] Sen, Souvik, et al. "Successive interference cancellation: Carving out mac layer opportunities" IEEE Transactions on Mobile Computing 12.2 (2013): 346-357.

[3] Jiang, Canming, et al. "Squeezing the most out of interference: An optimization framework for joint interference exploitation and avoidance" INFOCOM,

\section{Proceedings IEEE. IEEE, 2012.}

[4] Lv, Shaohe, et al. "Understanding the scheduling performance in wireless networks with successive interference cancellation" IEEE Transactions on Mobile Computing 12.8 (2013): 1625-1639.

[5] Liu, Runzi, et al. "SIC aware high-throughput routing in multihop wireless networks" Personal Indoor and Mobile Radio Communications (PIMRC), 2013 IEEE 24th International Symposium on. IEEE, 2013.

[6] Y. Hou, Ronghui, et al. "Hop-by-hop routing in wireless mesh networks with bandwidth guarantees" IEEE Transactions on Mobile Computing 11.2 (2012): 264277.

[7] Yuan, Di, et al. "On optimal link activation with interference cancelation in wireless networking" IEEE Transactions on Vehicular Technology 62.2 (2013): 939945 .

[8] Hou, R; Lui, KS; Baker, F; Li, J, "Hop-by-Hop Routing in Wireless Mesh Networks with Bandwidth Guarantees" IEEE Transactions on mobile Computing, vol. 11, NO. 2, FEBRUARY 2012

[9] J. Andrews, "Interference cancellation for cellular systems: a contemporary overview" IEEE Trans. Wireless Commun., vol. 12, no. 2, pp.19-29, 2005.

[10] C. Jiang, Y. Shi, Y. Hou, W. Lou, S. Kompella, and S. Midkiff, "Squeezing the most out of interference: An optimization framework for joint interference exploitation and avoidance" in Proc. IEEE INFOCOM,Mar. 2012, pp. 424-432.

[11] Salonidis, Theodoros, et al. "Identifying high throughput paths in 802.11 mesh networks: a model-based approach" Network Protocols, 2007. ICNP 2007. IEEE International Conference on. IEEE, 2007.

[12] Gao, Yan, Dah-Ming Chiu, and John Lui. "Determining the end-to-end throughput capacity in multi-hop networks: methodology and applications" ACM SIGMETRICS Performance Evaluation Review. Vol. 34. No. 1. ACM, 2006.

[13] Draves, Richard, Jitendra Padhye, and Brian Zill. "Routing in multi-radio, multi-hop wireless mesh networks" Proceedings of the 10th annual international conference on Mobile computing and networking. ACM, 2004.

[14] De Couto, Douglas SJ, et al. "A high-throughput path metric for multi-hop wireless routing" Wireless networks 11.4 (2005): 419-434.

[15] Jain, Kamal, et al. "Impact of interference on multi-hop wireless network performance" Wireless networks 11.4 (2005): 471-487. 